\title{
Analyzing Transitions of of Motivation and Learning Strategy with Ward Hierarchical Clustering
}

\author{
Dinh Thi Dong Phuong ${ }^{a,}{ }^{,}$, Hiromitsu Shimakawa ${ }^{\mathrm{b}}$ \\ a, ${ }^{\text {, }}$ Information and Engineering dept. \\ Ritsumeikan university, 1-1-1 Noji Higashi, Kusatsu, Shiga, Japan \\ *Corresponding Author: phuong@de.is.ritsumei.ac.jp
}

\begin{abstract}
Motivations and learning strategies of many student taking programming course change along a course. There are positive change, where students are motivated to the learning. There are also negative change, where students are demotivated to the learning. The programming educators should know the change, especially, negative change, to prevent a student demotivation.

Contextual inquiry is to get scenarios of students who already taken a programming course. The scenarios provides students motivation and learning strategies. Groups of students having similar motivation and learning strategies are achieved by classifying the scenarios using MSLQ. A persona is a virtual user with motivation and learning strategies representing a group of students as above. Web system is to get real learning behavior, called portfolios, of all of the students of the course. The paper uses Ward hierarchical clustering method to clusters the portfolios of every topics of the programming course. Clusters resulted from Ward method are mapped to personas found from scenarios. The result reveals which students belong to which personas at every topics. The paper clarifies the change of motivation of students belonging to a persona over topics of the course.
\end{abstract}

Keywords: programming course, motivation and learning strategy, contextual inquiry, scenarios, portfolios, Ward hierarchical clustering.

\section{Introduction}

Motivation of students taking programming courses are often very high at the beginning of the course. But, the motivation often decreases along the course, when the students encounter many difficulties which they cannot overcome. Educators should forecast the points when the motivation of students decrease to give timely support to them.

Assume the programming course are conducted with the same settings, e.g. curriculum and assignments contents, submission deadlines, etc., for two successive courses. From students of a course, the method gets scenarios of each of the student by contextual inquiry [3]. It also gets real learning behaviors, or portfolios, of every student using a learning support Web system. The scenarios bring about groups of students of similar motivation and learning strategies. Persona is a virtual user representing a group of similar students as above. Persona has motivation and learning strategies characteristics of the group it represents. Portfolios of every topics are used as input for Ward hierarchical clustering to get the students in each clusters. Each clusters resulted from Ward hierarchical clustering is mapped to each persona based on MSLQ[1]. As a result, we can find what students belongs to which persona as every topics. We also know the change of persona of the students along the course.

For the current course, the educators can understand the change of persona of a students using his or her portfolio until the current topics. From the understanding of the change of motivation and learning strategies of students belonging to personas from the last course, the educators can forecast the change of motivation of students toward the next topics.

\section{Student programing behaviors}

\section{$2.1 \quad$ Factors determining behaviors}

R.E.Slavin [2] says that "motivation is internal process that activates, guides and maintains behavior over time". A strong motivation to study programming would be a determining factor leading to the success of learning. Together with motivation, effective learning strategies are 
as important as the motivation to reach to achievements. MSLQ [1] is a good guideline to make questionnaires to understand components of motivation and learning strategies of a student in programming study. MSLQ not only provides descriptions of each component, but also enumerates sample questions.

For motivation, MSLQ lists up intrinsic, extrinsic, task value, expectancy, and affective components. Learners who have intrinsic components participate in target tasks because their goals are achievement of the tasks. They work because of reasons such as their challenge, curiosity, and mastery. For intrinsic components, MSLQ lists example questions like "In a class like this, I prefer course material that really challenges me so I can learn new things." Learners depending on extrinsic components participate in tasks because a learning task is the means to their goals. Components such as rewards, scores, and competition make them engage in the tasks. Task value components are determined by learner perceptions of the tasks in terms of interest, importance, and utility. Expectancy components refer to learner beliefs that their efforts will results in good outcomes. Affective components are related to anxiety for tests, grading for credits, and so on. For example, since they worry about tests, they make efforts.

A learning strategy is a personal approach to understand information and solve problems. Learning strategies consists of resource management strategies and selfregulation strategies. The resource management relates to the learner ability to understand the usefulness and effectiveness of given resources for the learning. As for resource management, MSLQ enumerates study environment strategies regarding to setting of study places, peer learning strategies corresponding to collaboration with peers, and help seeking strategies to get supports from others including peers and instructors. Self-regulation strategies work in the process whereby learners systematically direct their thoughts, feelings, and actions toward the attainment of their goals [4]. Yukselturk [5] states self-regulation learning strategies are strong predictor for success in on-line courses. Self-regulation strategies in MSLQ involve cognitive strategies, meta-cognitive strategies, time strategies, and effort regulation strategies. Cognitive strategies refers to methods to learn, such as how to remember new concepts, to organize them, and to understand them, while meta-cognitive strategies correspond to methods to tune and adjust cognitive strategies. Time strategies plan their study time. Effort regulation strategies control their efforts in the face of distractions and uninteresting tasks.

\subsection{Context}

Some students are eager for programming, while others have no interest in it. Every learner has a certain state in a specific time. In a learning process, students receive stimuli in her education environment. Suppose a student who stays in a certain state. When she receives a stimulus, she takes an action against the stimulus, depending on the current state in her environment. Her state changes into a new one after the action.

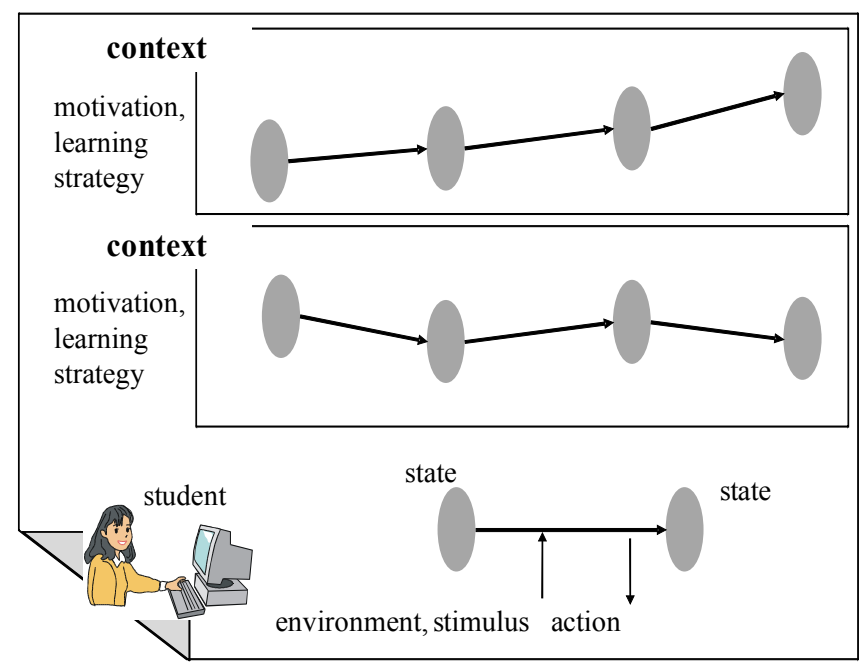

Figure 1. Motivation and learning strategy shapes the context

A context is a chain of states which change according to stimuli. Figure 1 visualizes the concept of a context. Since the chain of states in a context results from certain motivation and learning strategy, the analysis of a context reveals the motivation and learning .

We use the contextual inquiry to get contexts in the learning of past students through interviews. The contexts are stated in scenarios which are outcomes of the interviews. Each scenario expresses many transitions of states of a student. There are many contexts described in a scenario. Classifying similar scenarios into several groups, we obtain motivation and learning strategies of students who belong to each of the groups. A persona is assigned to each group. A persona is a virtual student representing motivation and learning strategies of students in one group. MSLQ works as a good guideline to classify students from the view point of motivation and learning strategies stated in the scenarios.

\subsection{Student portfolio}


Motivation and learning strategy are expressed to the outside through behaviors reflecting contexts. Good facilitation tools for programming learning can record the behaviors of the students. The records indicate, for example, when a student studies programming, what assignments she solves, where she solves it, and the articles she refers to during the learning process.

Table 1 shows an example of a portfolio of 5 students from $\boldsymbol{a}$ to $\boldsymbol{e}$. It represents 7 features of each student. The learning time is the time they make access to the programming practice support web site which gives assignments, sample codes, guidance, and so on. The submissions are the total number of source codes submitted to the servers. The compilation frequency is the average compilation times of a source code over a specific time period. The compilations are the average compilation times until they submit a source code. The viewing check-item indicates the times the student refers the check items, which show explanation and guidance for students to achieve a good source code.

Obviously, if a student works hard to achieve good source code, her learning time would be long. If a student has a willing mind of challenge and exploration, she would solve many assignments. If a student spends little time to make source codes for difficult assignments, the codes would not be the good one, which gets low score. Responses to stimuli in specific contexts depend on the motivation and learning strategies of the students. Progressive learning behavior represented in the portfolio does represent motivation and learning strategy of each student.

Table 1. Portfolio of students

\begin{tabular}{|l|c|c|c|c|c|c|c|}
\hline $\mathbf{X}$ & $\begin{array}{l}\text { Learning } \\
\text { time }\end{array}$ & $\begin{array}{l}\text { Submi- } \\
\text { ssions }\end{array}$ & $\begin{array}{l}\text { Compilation } \\
\text { frequency }\end{array}$ & $\begin{array}{l}\text { Compi- } \\
\text { lations }\end{array}$ & $\begin{array}{l}\text { Viewing } \\
\text { check-item }\end{array}$ & $\begin{array}{l}\text { Referring } \\
\text { samples }\end{array}$ & Score \\
\hline a & 71.97 & 32.17 & 1.25 & 16.8 & 4.89 & 5.0 & 5.7 \\
\hline b & 110.01 & 38.96 & 1.15 & 26.4 & 4.47 & 7.0 & 8.2 \\
\hline c & 113.78 & 41.78 & 1.3 & 27.2 & 5.06 & 5.06 & 8.9 \\
\hline d & 92.6 & 30.27 & 0.78 & 22.4 & 3.02 & 4.02 & 5.5 \\
\hline e & 49.01 & 36.66 & 1.95 & 10.4 & 7.67 & .67 & 4.2 \\
\hline
\end{tabular}

\section{Persona estimation of a student}

\subsection{Scenario}

In the proposed method, the classification of student groups is essential to achieve a good decomposition of a portfolio matrix into the product of a weight matrix and a feature matrix. Especially, features of motivation and learning strategies to distinguish each group play vital roles. They not only work as criterion for the classification, but also represent the degree of each student in portfolio belonging to them.

With questionnaire methods, it is hard to get real and practical hints for the classification. Since the students who give answers to the questionnaire may not have real experiences for conditions assumed in questions, questionnaire methods prevent collection of truthful information from learners. On the other hand, the contextual inquiry is a good way because the students join the interviews freely talk about their real experiences, from which, real learning contexts are expressed. The contexts reveal hints for features.

The contextual inquiry method insists to delve into learning processes of the students with interviews among themselves. Every detail of behaviors of students in specific conditions is clarified as an interview goes on. Suppose the student mentions help from her friend to solve the assignment, when an interviewer who experienced the same programming course, asks how she solves an assignment. The interviewer asks why the help occurs, how the help takes place, and how the interviewee feels after the help. The further the interviewer delves into the context, the clearer image of the learning process the interviewer obtains. A scenario summarizes what the interviewee has experienced. The scenario expresses contexts, which consist of sequences of actions and feelings toward maters occurring in the learning process in specific conditions of the student.

\subsection{Persona}

As Cooper states, scenarios of learners similar in their characteristics can be compiled into a scenario for a persona. A persona is a virtual learner with a scenario stating its behaviors in specific contexts. After obtaining scenarios of contexts, we know motivation and learning strategy of every student, referring to MSLQ. Scenarios similar in motivation and learning strategy are put into a group. A persona represents motivation and learning strategy of a group. A scenario of a persona presents typical learning contexts of students corresponding to the persona.

Personas and their scenarios make the designer of programming course grasp a single image of members of a student group in advance. In the course time, if the supervisor knows what persona the student belongs to, or in other words, her motivation and learning strategy in a specific context, the 
supervisor would give more appropriate supervisions to the student.

Moreover, the number of students join the course are often very big, because programming courses are usually compulsory ones in computer science education programs. The students are divided into separate classes where a teacher and some teaching assistants take in charge of supervisions. It causes the variance of teaching among the classes. The variance should be avoided to bring a fair education to all of the students taking the course. In each class of about 40 students, the diversity of the students is also the mater preventing the persons in charge from giving a fair education. Persona contributes minimize the variance among the classes as well as covering the diversity of students in each class.

\section{Persona motivation and learning strategies characteristics}

\subsection{Obtaining persona}

The contextual inquiry method is used to get scenarios expressing learning contexts of the students. We let students having finished the last programming course conduct interviews with each other to describe their contexts. The good time for the interview is at the beginning of right next semester after the semester of the programming course. The students have enough break after the programming course. If we ask them to do the interview right after the programming course, many complaints would fill up the interviews because the students have to make a lot of efforts in the programming course. If the interviews are conducted after a too long break, they would lose truthful contexts.

The inquiry topic is programming motivation. Following is one snippet of one interview of student $\mathrm{R}$ and student $\mathrm{E}$.

$R$ : What makes you incline to programming?

E: I regard programming as a challenge. When I have solved a tough assignment, I get a strong sense of achievement.

$R$ : When you face a tough one, you might sometimes find no way to solve it. Have you ever run into such a situation?

E: Yes, many times. In such a case, I will repeat to check sample codes in the textbook. Sometime, I try easier assignments in the same section.

$R$ : What will you do, if you cannot get anything from them?

E: I will search Web pages explaining similar matters using the Internet. I do not prefer to be supervised by TAs, because I feel lost in the challenge.

$R$ : But, they give you clues, even an encouragement, sometimes.

E: I am not pleased, even if they encourage me. But, when they give high grades to my codes, I get satisfactions.
Scenarios are created from the interviews of 46 students. The scenarios are classified using MSLQ with three range of motivation and learning strategy: strong, weak, and not have. The result reveals 5 groups of students similar in motivation and learning strategies. Each group is named as its representative characteristics.

The industrious group, consisting of 13 students, are ones with high will to learn. They understand the more they engage in the study, the more they can get knowledge and skills. Some of them want their high ability to be praised by supervisors or friends. This group, represented by persona challenging persona, is specified to have strong intrinsic, expectancy, and task value components, along with weak extrinsic ones. Moreover, challenging persona knows how they should study to improve their abilities. They know to utilize various resources such as explanations by teaching assistants (TAs) and sample codes in the Internet. It reveals that challenging persona has learning strategies to achieve a good result.

The easy-going group, containing 19 students, understands the compulsory programming subject brings many benefits to them. Their learning purpose is to get a credit. Their attitudes to the learning are not stable. As far as they can go on with assignments easily, they are eager to study. But, for assignments which seem hard to solve, they tend to put them aside, because they do not believe their ability. They often stick to specific resources such as TA helps. They do not know utilizing many kinds of resources. We refer to this group as persona easygoing persona. We regard easygoing persona has weak intrinsic, and strong extrinsic, weak task value, and strong affective components. easygoing persona has no learning strategy.

The obligated persona, with 7 students, learns hard. However, it does not have any interest in the learning. It obeys the course settings well, such as, submissions before deadlines. It just tries to fulfill all the course requirements. It does not show any creative in the learning. It has little task value, little self efficacy.

The demanding persona, with 2 students, has little interest in the learning. It learns only with the support of TAs. Its always demands helps and answers. It is afraid of deadline very much.

The unwilling persona does not like programming learning but have to, because of the compulsory subject. They learn only at school with help of supervisors. Different from industrious persona and easygoing persona, unwilling persona does not show any will to learn. They absolutely have no interests in the learning. Unwilling persona has no intrinsic component. They may quit the course any time. 


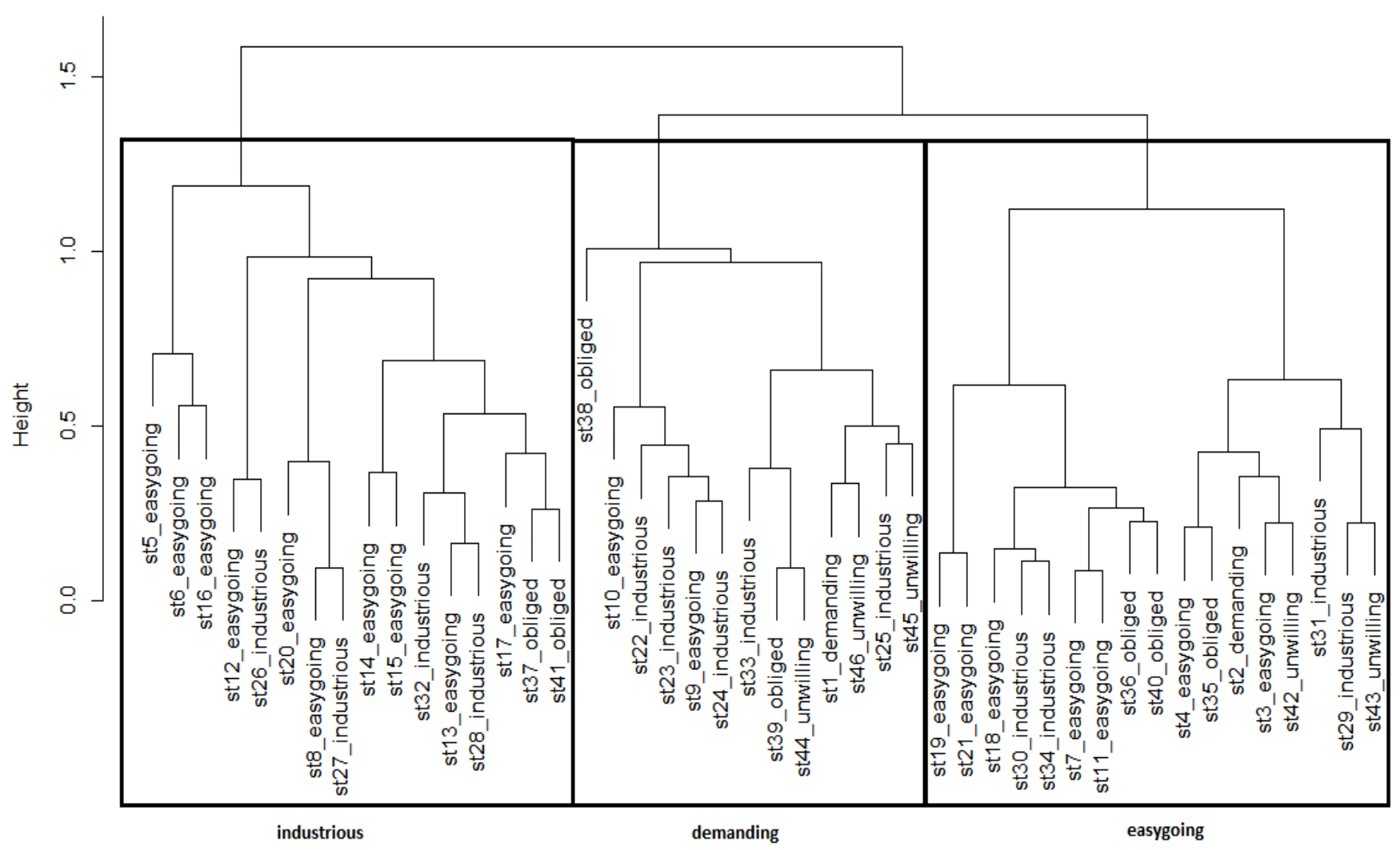

Figure 2. Portfolios Clusters of week 7

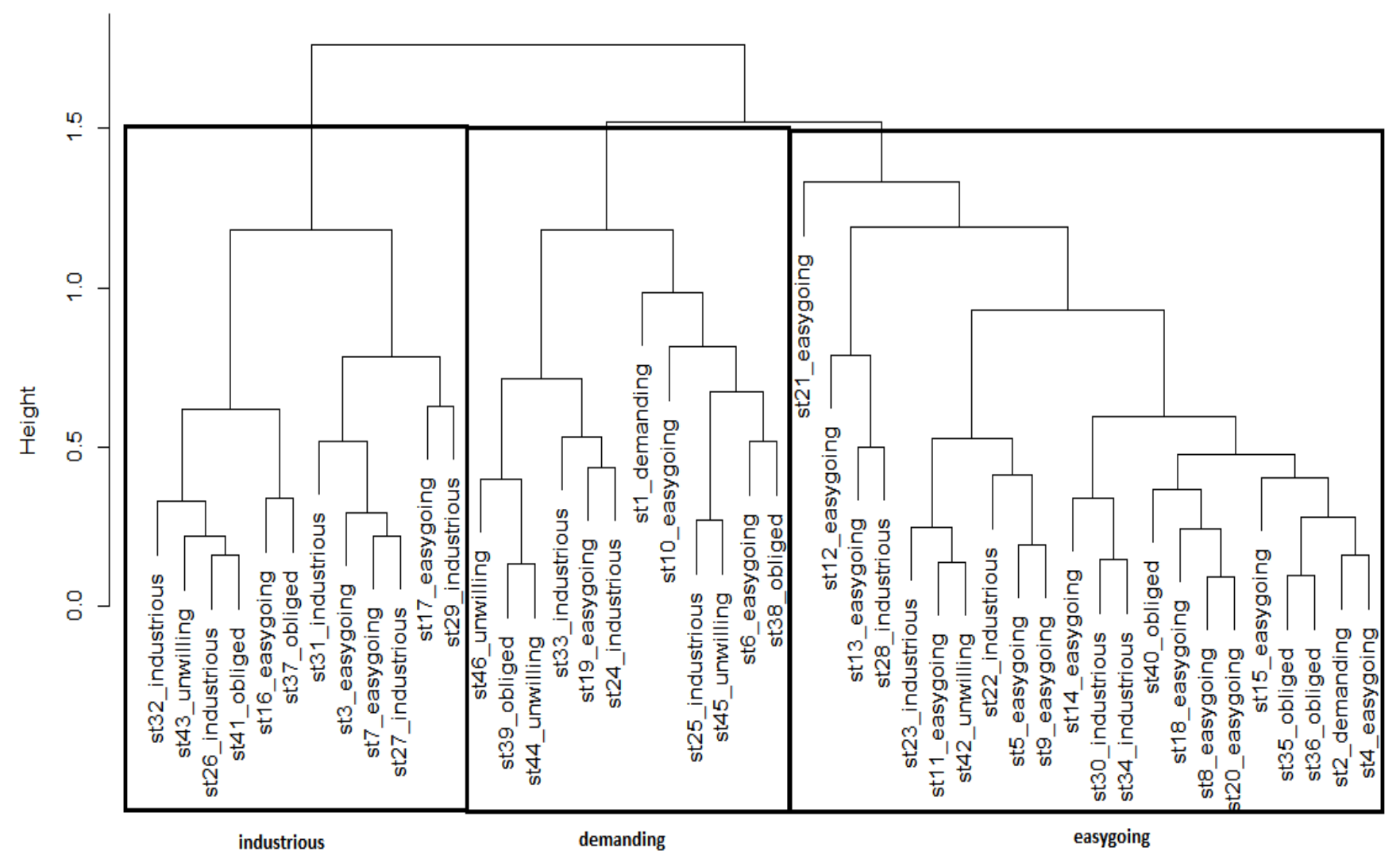

Figure 3. Portfolios Clusters of week 8 


\subsection{Students clusters from portfolios}

Real learning behaviors of the 46 students brings about their portfolios of every week. The portfolios consist of their learning time, times of log-in to the Web system, submissions of advanced assignments, submissions in the day just before the deadline, total clicks on the pages of the Web system, score of assignments of the week.

Let us consider two students in the left most of the left cluster of week 7, industrious cluster. They are denoted as st5_easygoing and st6_easygoing. In this week, they are in the industrious cluster. In other words, they have similar motivation and learning strategies in week 7 . However, in week 8, student st5_easygoing fall into the right most cluster, the easygoing one, while student st6_easygoing fall into the middle cluster., demanding cluster. They are not in the same cluster in week 8 .

The industrious cluster of week 7 has total 16 students. Among them, 7 student (st16_easygoing, st26_easygoing, st27_industrious, st32_industrious, st17_easygoing, st37_obligated, st41_obligated) belong to the industrious cluster in week 8. 8 other students (st5_easygoing, st12_easygoing, st20_easygoing, st8_easygoing, st14_easygoing, st15_easygoing, st13_easygoing, st28_industrious) belong to the easygoing cluster. The industrious cluster in week 7 go into two different clusters in week 8 . A half number of students of the left most cluster in week 7 change their motivation and learning strategies from week 7 to week 8 .

\section{Conclusions}

The paper analyzes the change of motivation and learning strategies of students learning programming. At the current phase, Ward method is used to cluster students. The weight which represents how much a student belongs to a cluster cannot be clarified with Ward method. We are using non-negative matrix factorization to understand the weight.

\section{References}

(1) P.R.Pintrich, D.A.F.Smith, T.Garcia, and W.J.McKeachie, A Manual for the Use of the Motivated Strategies for Learning Questionnaire(MSLQ), National Center for Research to Improve Post SecondaryTeaching and Learning, 1991.

(2) R.E.Slavin, Educational psychology: Theory and Practice, 10th, Edition, Pearson, pp.317, 2006.
(3) A.Cooper, The Inmates are Running the Asylum: Why High Tech Products Drive Us Crazy and How to Restore the Sanity, Pearson Education, 2004.

(4) D.H.Schunk, and B.J.Zimmerman, Self-regulated learning: From teaching to self-reflective practice, Guilford Press, 1998.

(5) E.Yukselturk, S.Bulut, Predictors for Student Success in an Online Course, Educational Technology \& Society, Vol.10, Issue 2, pp.71$83,2007$. 